\title{
Current Challenges and Applications for Adaptive User Interfaces
}

\author{
Victor Alvarez-Cortes, Víctor H. Zárate, Jorge A. Ramírez Uresti and \\ Benjamin E. Zayas \\ Instituto de Investigaciones Eléctricas; Tecnológico de Monterrey \\ México
}

\section{Introduction}

In this chapter we present the current advances in the field of adaptive user interfaces, analysing the different research efforts, the challenges involved as well as the more recent and promising directions in this field. Initially, we introduce the foundations of adaptive user interfaces, also referred in technical literature as Intelligent User Interfaces (IUIs), then we move to explore the motivation and rationale for their use, and finally we discuss the challenges they currently have to deal with. In this context, IUIs are presented as a multidisciplinary field, with relevant research and cross-fertilized ideas derived from different areas, however special emphasis is put on the approaches taken by three core disciplines: Artificial Intelligence (AI), User Modelling (UM) and Human-Computer Interaction (HCI). After providing the foundations for IUIs, an in-depth revision for each approach is presented including the most recent findings in models, algorithms and architectures for adaptive user interfaces.

Although, adaptive user interfaces are considered a recent research field, this chapter is enriched with a state-of-the-art of IUIs applications. The material included presents the most relevant developed IUIs applied in different real domains either as a research prototype or as a complete system. A methodological analysis of these systems is presented, contrasting its advantages, limitations and domain-dependence for its success and acceptance by users. The analysis aims to uncover common principles for effective IUI design. Also, this chapter details our proposed taxonomy which is applied for the comparison of the different IUIs systems.

Finally, the chapter presents the gaps left by the approaches under analysis and concludes with a discussion of the challenges currently open, presenting a number of possible future research directions.

Las interfaces de usuario para los sistemas de computación han cambiado mucho en los últimos 20 años. Las primeras interfaces basadas en texto que utilizaban la línea de comando para acceder a los recursos del sistema operativo, han sido sustituidas por interfaces gráficas que son manipuladas a través de dispositivos de entrada como el teclado y ratón. En la actualidad, la interfaces buscan ser más intuitivas al usuario al presentar elementos gráfico de fácil asociación con elementos reales mediante el uso de metáforas (Dix et al., 2003). 
Un paradigma de interacción utilizado de manera extensa en los sistemas operativos actuales es el uso de múltiples ventanas para presentar información, el uso de iconos para representar elementos del entorno como son carpetas, archivos, dispositivos, etc, junto con el uso de menús y botones, que faciliten la interacción con el sistema. A este paradigma se le conoce como WIMP (Windows, Icons, Menus, Pointers) desarrollada por Xerox PARC en los 80`s, y utilizado inicialmente por las computadoras Apple Macintosh y actualmente disponibles en otros sistemas como Microsoft Windows, OS/Motif, Risc OS y X Window System (Shneiderman \& Plaisant, 2004). Sin embargo, aún con estos avances y la funcionalidad ofrecida por las interfaces de usuario de los sistemas actuales, la mayoría de ellas aún siguen siendo limitadas en cuanto al manejo de las diferencias que existentes entre los diversos usuarios de la interfaz, quedando clara que existe una limitación en el desarrollo de sistemas que puedan ser personalizados y adaptados al usuario y al entorno.

Las Interfaces Inteligentes de Usuario (IUI, por sus siglas en inglés) es un sub-campo de HCI y tiene como objetivo mejorar la interacción humano-computadora mediante el uso de nueva tecnología en dispositivos de interacción, así como a través del uso de técnicas de inteligencia artificial, que le permitan exhibir algún tipo de comportamiento inteligente o adaptivo.

\section{Uso de las IUIs}

Las IUIs tratan de resolver algunos de los problemas que las interfaces tradicionales, llamadas de manipulación directa (Shneiderman, 1997) no pueden afrontar.

- Crear sistemas personalizados: No existen dos usuarios que sean iguales y cada uno tiene diferentes hábitos, preferencias y formas de trabajar. Una interfaz inteligente puede tomar en consideración estas diferencias y proporcionar métodos personalizados de interacción. La interfaz conoce al usuario y puede usar ese conocimiento para establecer el mejor medio de comunicación con el usuario.

- Problemas de filtrado y exceso en información: Tratar de encontrar la información necesaria en una computadora o en Internet puede resultar una tarea complicada. Aquí una interfaz inteligente puede reducir la cantidad de información relevante en grandes base de datos. Al filtrar información irrelevante, la interfaz puede reducir la carga cognitiva del usuario. Adicionalmente una IUI puede proponer nuevas y útiles fuentes de información desconocidas al usuario.

- Proporcionar ayuda para nuevos programas: Los sistemas de información pueden llegar a ser muy complicados de usar al inicio. Es común que conforme el usuario empieza a entender las funcionalidades del programa, nuevas versiones o actualizaciones aparecen, incluyendo nueva funcionalidad. En esta situación un sistema inteligente de ayuda puede detectar y corregir usos incorrectos o subóptimos para realizar una tarea, explicar nuevos conceptos y proporcionar información para simplificar las tareas.

- Hacerse cargo de tareas por el usuario: Una IUI puede ver que es lo que está haciendo el usuario, entender y reconocer su intento y ocuparse de la ejecución completa de ciertas tareas, permitiéndole al usuario enfocarse en otras.

- Otras formas de interacción: En la actualidad los dispositivos más comunes de interacción son el teclado y el ratón. Una línea de investigación de IUI conocida como interfaces multimodales investiga nuevas formas de interacción a través de 
otras modalidades de entrada y salida para interactuar con el usuario. Al proporcionarse múltiples formas de interacción, la gente con discapacidades podrá utilizar sistemas complejos de cómputo.

\section{Definición y áreas relacionadas}

A través de los años numerosas definiciones de inteligencia han surgido para definir sistemas y comportamientos, sin embargo no existe un consenso sobre qué se debe considerarse inteligente. No obstante, la mayoría de las definiciones mencionan la habilidad de adaptación ( aprender y lidiar con nuevas situaciones), la habilidad para comunicarse y la habilidad para resolver problemas (Russell \& Norvig, 2003).

Una interfaz "normal" es definida de manera simple como la comunicación entre un usuario (humano) y una máquina (Meyhew, 1999). Una extensión de esta definición para una interfaz inteligente es que utiliza algún tipo de componente inteligente para llevar a cabo la comunicación humano-computadora. Por eso también se les conoce como interfaces adaptivas, porque tienen la habilidad de adaptarse al usuario, comunicarse con él y resolverle problemas. Una definición más formal es: Las interfaces inteligentes de usuario buscan mejorar la flexibilidad, usabilidad y poder de interacción humano computadora para todos los usuarios. Al hacerlo, explotan el conocimiento de los usuarios, las tareas, herramientas y contenido, así como los dispositivos para soportar la interacción con diferentes contextos de uso (Maybury99].

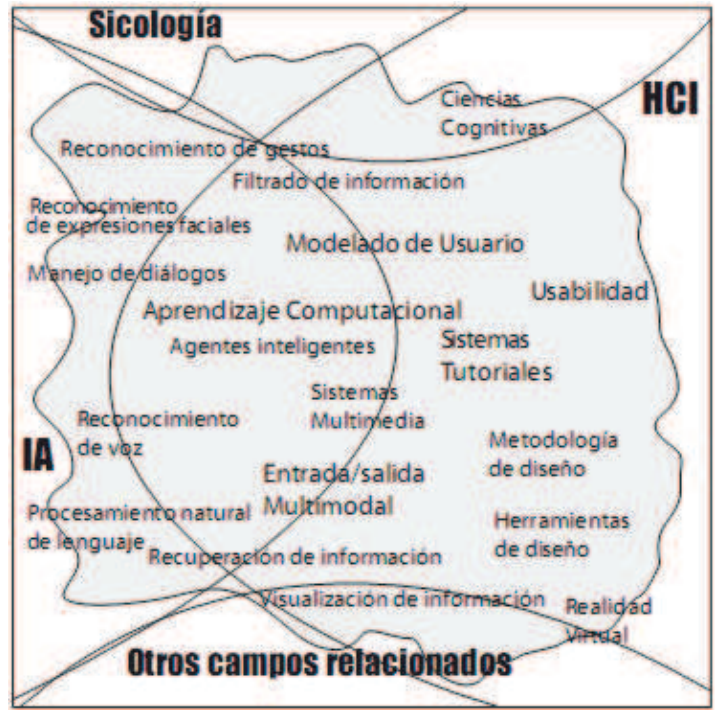

Figure 1. IUIs y sus diferentes disciplinas

Debido a que la adaptación y la resolución de problemas son temas centrales de investigación en inteligencia artificial, muchas IUIs están fuertemente orientadas al uso de técnicas de IA, sin embargo no todas las IUIs tienen capacidad de aprendizaje o resolución de problemas. Muchas interfaces que se denominan inteligentes se enfocan al aspecto del canal de comunicación entre el usuario y el sistema (máquina) y generalmente aplican 
técnicas nuevas de interacción como procesamiento de lenguaje, seguimiento de mirada (gaze tracking) y reconocimiento facial. Lo cierto es que muchos campos de investigación tienen influencia en las IUIs tales como la sicología, ergonomía, factores humanos, ciencias cognitivas y otras como se muestra en la figura 1.

Una de las propiedades más importantes de las IUIs es que son diseñadas para mejorar la comunicación entre el usuario y la máquina.

No importa mucho que tipo de técnica sea utilizada para conseguir esta mejora. Una lista de varios tipos de técnicas que son usadas en las IUIs son:

- Tecnología de entrada inteligente: se refiere al uso de técnicas para obtener la entrada del usuario. Estas técnicas incluyen lenguaje natural (reconocimiento de habla y sistemas de diálogo), seguimiento y reconocimiento de gestos, reconocimiento de expresiones faciales y lectura de labios.

- Modelado de usuario: aquí se incluyen las técnicas que le permiten a un sistema mantener o inferir conocimiento acerca de un usuario basado en la entrada de información recibida.

- Adaptividad de usuario: comprende todas las técnicas que permiten que la interacción humano-computadora sea adaptada a diferentes usuarios y diferentes situaciones de uso.

- Generación de explicaciones: comprende todas las técnicas que permiten que la interacción humano-computadora sea adaptada a diferentes usuarios y diferentes situaciones de uso.

- Personalización: Para poder personalizar las IUIs normalmente incluyen una representación del usuario. Estos modelos de usuario registran datos acerca del comportamiento del usuario, su conocimiento y habilidades. Nuevo conocimiento del usuario se puede inferir basado en las entradas y el historial de interacción del usuario con el sistema.

- $\quad$ Flexibilidad de Uso: Para ser flexibles muchas IUIs utilizan adaptación y aprendizaje. La adaptación toma lugar en el conocimiento almacenado en el modelo del usuario al hacer nuevas inferencias al usar la entrada actual. El aprendizaje ocurre cuando cambia el conocimiento almacenado para reflejar las nuevas situaciones encontradas o nuevos datos.

\section{Inteligentes y Sistema Inteligentes}

Con frecuencia se comete el error de confundir lo que es una IUI con lo que es un sistema inteligente. Un sistema que muestra alguna forma de inteligencia no necesariamente es una interfaz inteligente. Existen muchos sistemas inteligentes con interfaces de usuario muy simples que no son inteligentes. Asimismo el hecho de que un sistema tenga una interfaz inteligente no nos dice nada acerca de la inteligencia que existe en el sistema. Ver figura 2. Desafortunadamente la frontera entre un sistema y su interfaz de usuario no siempre es tan claro como en la figura y muchas veces la tecnología utilizada en una IUI también es parte del sistema, o la IUI representa en realidad la totalidad del sistema. Asimismo muchas veces se pone poco énfasis en la interfaz y su separación para ser desarrollada por un grupo especializado en interfaces y ergonomía, no siempre son una realidad. A menudo al desarrollar un sistema se le da mayor importancia a la parte interna y de algoritmos, dejando la parte de la interfaz como en componente más del sistema (Hook, 1999). 


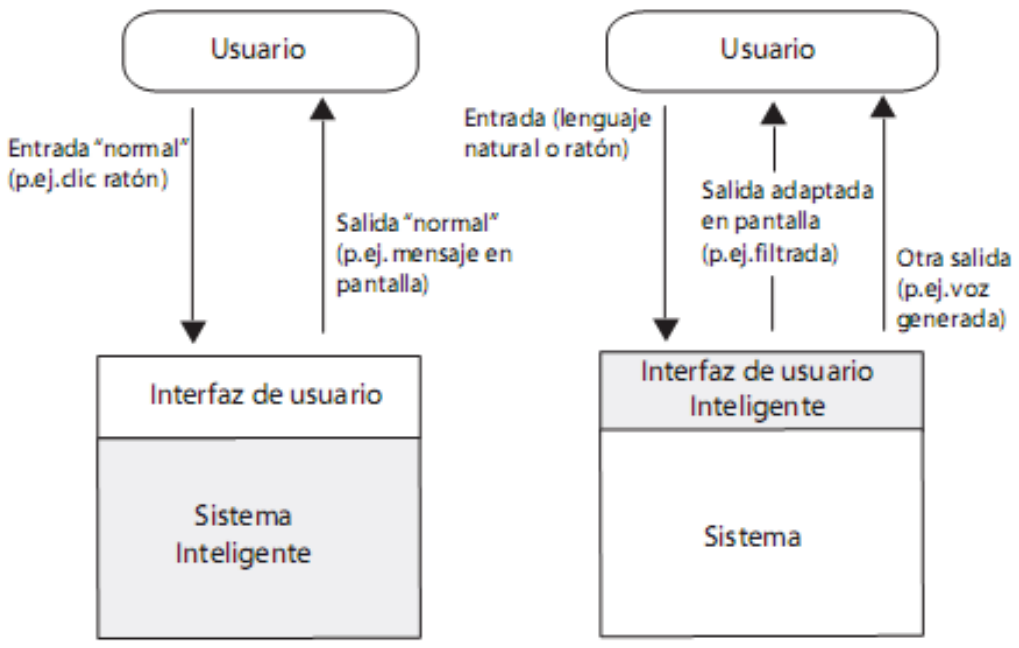

Figure 2. Sistema inteligente vs Interfaz inteligente

\section{Beneficios y críticas}

El campo de las IUIs en ninguna manera se encuentra maduro y aún existen problemas abiertos que deben resolverse. Quizá esta se una de las razones por la cuál las IUIs (gracias a la IA) ha recibido escepticismo de una parte de HCI. El problema central radica en que las IUIs violan principios de usabilidad aceptados dentro del desarrollo de sistemas de manipulación directa. Los trabajos de Maes en el MIT (Maes, 1994) y otro de Wernn (Wernn, 1997) advierten sobre el potencial de las interfaces inteligentes y sus capacidades, ponen de manifiesto los puntos que deben ser resueltos antes de que las IUIs sean aceptadas y usadas de manera extendida.

Para Shneiderman (Shneiderman,1997) un sistema adaptivo es impredecible y menos transparente que una interfaz tradicional. Si un sistema puede adaptar su respuesta y no da la misma salida dos veces ante la misma entrada, el sistema entonces se vuelve impredecible. Otro problema similar es el control sobre la interfaz. Las posibilidades son si el usuario toma el control y decide la siguiente acción a realizarse o si el sistema de manera autónoma con base a su conocimiento parcial toma el control y decide la siguiente acción. Esta situación ha sido abordada desde diferentes enfoques dando lugar a especializaciones dentro de las interfaces de usuario inteligentes.

Las interfaces adaptables permiten que el control lo tenga el usuario y sea él quien dirija y controle la adaptación a través de opciones para poder personalizar y adaptar la interfaz. En el otro lado encontramos las interfaces adaptivas donde el sistema tiene la inteligencia suficiente y realiza la evaluación del estado para llevar a cabo algún tipo de adaptación sin la intervención del usuario. Un esquema de interacción que ha recibido aceptación es el de iniciativa mixta o combinada, donde se comparte le interacción entre el usuario y el sistema (Armentano, 2006). Una discusión más extensa entre expertos del área de interfaces sobre las ventajas y desventajas entre las IUIs y la interfaces de manipulación directa la encontramos en el trabajo de Birnbaum (Birnbaum, 1997). 


\section{Aprendizaje Computacional en las IUls}

El uso de técnicas de inteligencia artificial para diseñar IUIs ha sido un enfoque que ha sido aceptado en la comunidad de interfaces y aplicado a diferentes dominios y aplicaciones.

Las técnicas reportadas en la literatura van desde los tradicionales sistemas de producción (basados en reglas) hasta técnicas más modernas como son planificación y modelos probabilísticos gráficos (Horvitz, 1998). Técnicas más recientes de IA también se han utilizado en las IUIs tales como agentes autónomos (Rich \& Sidner, 1996), (Eisenstein \& Rich, 2002).

Todas estas técnicas han sido utilizadas para generar diferentes grados de inteligencia o adaptabilidad y aprovechar el conocimiento que se tiene del usuario y sus tareas para proporcionar una mejor interacción y experiencia de uso del sistema.

Las técnicas y algoritmos de aprendizaje computacional (Machine Learning (ML) han tenido un rápido desarrollo ganando aceptación dentro de la comunidad de AI. El aprendizaje computacional ha sido aplicado con relativo éxito en varias dominios, principalmente en aplicaciones Web para colectar información y minar datos sobre el historial de interacción y de navegación de los usuarios (Fu, 2000). Asimismo el aprendizaje ha sido utilizado como un medio para inferir modelos de usuario (Stumpf et al., 2007) basado en datos pasados con el objeto de descubrir patrones desconocidos y adaptar el comportamiento de la interfaz.

En términos de ML, una interfaz inteligente puede ser conceptualizada como "un componente de software que mejora su capacidad para interactuar con el usuario mediante la construcción de un modelo basado en la experiencia parcial con ese usuario" (Langley, 1999). Esta definición muestra claramente que una interfaz inteligente está diseñada para interactuar con usuarios reales y humanos. Aún más, si la interfaz debe ser considerada como inteligente, entonces debe mejorar su interacción con el usuario al pasar el tiempo, considerando que una simple memorización de esas interacciones no es suficiente, sino que la mejora debe provenir como resultados de una generalización en experiencias pasadas para establecer nuevas interacciones con el usuario.

Es posible identificar dos amplias categorías de interfaces inteligentes dentro del enfoque de ML, las cuales difieren en el tipo de retroalimentación que debe proporcionar el usuario:

- Informativas: Este tipo de interfaz trata de seleccionar o modificar información para el usuario al presentar sólo aquellos elementos que el usuario pueda encontrar interesantes o útiles para la tarea que está realizando. Los ejemplos más comunes son los sistemas de recomendación de productos (i.e la librería en línea Amazon.com) y filtros de noticias, donde se dirige la atención de los usuarios dentro de un amplio espacio de opciones. En este tipo de sistemas la retroalimentación del usuario generalmente incluye marcar las opciones de recomendación como deseables y no deseables y evaluarlas asignando alguna puntuación. Sin embargo esta clase de interfaces distrae la atención de la tarea central, pues el usuario debe proporcionar retroalimentación. Se reporta en la literatura la existencia de métodos menos intrusivos para obtener retroalimentación al observar el proceso de acceso (Sugiyama, 2004).

- Generativas: Este tipo de interfaces están enfocadas principalmente a la generación de alguna estructura útil de conocimiento. Aquí se incluye programas para la preparación de documentos, hojas de cálculo, así como también sistemas para planificación y configuración. Estas áreas soportan un tipo de retroalimentación mejorada ya que el usuario puede no sólo ignorar alguna recomendación, sino 
sustituirla por otra. Los tipos de retroalimentación están amarrados a los tipos de interacción que soporte la interfaz. Varios sistemas requieren que el usuario corrija acciones no deseables, lo cual es un problema de interrupción, sin embargo sistemas recientes incorporan esquemas menos instrusivos mediante la observación de las acciones del usuario. (Franklin et al., 2002).

También se reporta en la literatura que minar los registros del uso en Web es un enfoque factible para construir interfaces Web adaptivas . El historial de acceso Web constituye una fuente abundante de información que permite experimentar con conjuntos de datos reales. Los sistemas Web adaptivos le facilitan al usuario la navegación al proporcionarle accesos directos a sitios de manera personalizada. Durante el proceso de adaptación, los datos de acceso del usuario son la fuente central de información y es utilizada para construir el modelo del usuario, que refleja el patrón que el sistema infiere para los usuarios y describe varias características de los mismos. La minería de datos usando reglas de asociación se ha utilizado para minar el historial de navegación de sitios Web y también para proponer o sugerir nuevas ligas basada en un filtrado colaborativo.(Mobasher et al., 2001).

\section{Modelado del usuario en la IUls}

La interfaz de usuario y el modelado del usuario (UM) pueden ser vistos como como dos lados de un mismo componente. El modelo de usuario consiste en los algoritmos que son implementados en los componentes de software mostrando el concepto de personalización desde la perspectiva del sistema. Por otro lado, la interfaz inteligente es la interfaz gráfica (GUI) y le presenta al usuario un contenido generado por el UM, mostrando la personalización desde el punto de vista del usuario como se muestra en la figura 3.

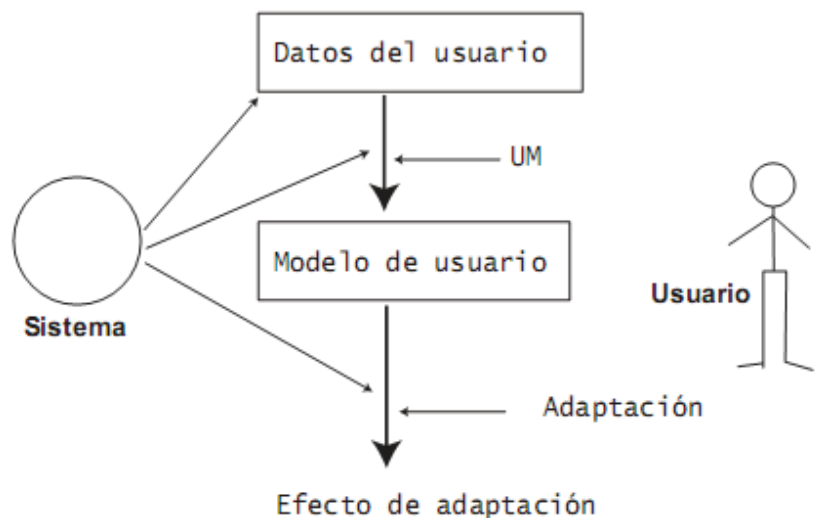

Figure 3. Dos perspectivas del modelos del usuario

Las aplicaciones de modelado de usuario pueden ser definidas como aplicaciones donde "usuarios con diferentes objetivos, intereses, niveles de experiencia, habilidades y preferencias pueden adecuar a la medida el comportamiento del programa a necesidades individuales generalmente mediante el uso de un perfil de usuario" (Kobsa, 1994)

El propósito del UM es colectar, procesar y mostrar datos adaptados al nivel de la interfaz mediante una recopilación de conocimiento de información de dos tipos. 
- Implícita: Esta recopilación de datos involucra examinar archivos de registros como historiales de navegación o los "cookies" en un navegador (Fu, 2000). El descubrimiento de patrones en los datos permite el filtrado de contenidos y su direccionamiento $\mathrm{y}$ entrega basada en suposiciones hechas sobre el comportamiento del usuario. Por ejemplo, si un usuario regularmente revisa ciertos elementos (ítems) particulares, el UM identificará el patrón y puede alterar la interfaz para desplegar esos elementos y asociar el contenido en una pagina Web de acceso al usuario. Esta es una técnica automatizada de UM que no requiere de ninguna retroalimentación directa del usuario para identificar un patrón de comportamiento y modificar el contenido de acuerdo a éste.

- Explícita: La captura de datos explícitos involucra el análisis de datos metidos por el usuario, proporcionando información acerca de sus preferencias mediante el llenado de un perfil de usuario. Ejemplos de estos datos son la edad, sexo, ciudad, historial de compras, preferencias sobre contenidos y distribución de la información en pantalla. Esta técnica también se conoce en el área de modelado como una técnica UM informada. Esta técnica requiere la entrada de datos por parte de los usuarios y un mecanismo para reconocer patrones en las preferencias de los usuarios con el objeto de modificar la interfaz basada en esas preferencias.

Los modelos de usuario pueden ser utilizados para crear servicios personalizados mediante la adaptación a necesidades individuales usando información o técnica de filtrado colaborativo (Carenini, 2003). Los usuarios pueden tomar control sobre su interacción al elegir contenidos de manera explícita basada en perfiles de usuarios o a través de sistemas de recomendación que establezcan asociaciones entre un historial de compra individual o de navegación con el de otros usuarios similares (Kirsh-Pinheiro et al., 2005).

Una interfaz adaptiva presenta los modelos de usuario al nivel de la interfaz al desplegar contenidos personalizados (Eirinaki \& Vazirgiannis, 2003). Asimismo los usuarios también tienen la oportunidad de interactuar directamente con el modelo de usuario cuando lo crean o al editar los perfiles. El modelado de usuario y la interfaz adaptiva esencialmente presentan vistas personalizadas de contenidos que pueden ahorrar tiempo para localizar información o guiar a los usuarios a contenidos disponibles y desconocidos al usuario. En resumen, el UM es una alternativa sólida para la presentación de contenidos personalizados y es considerado una herramienta útil para el usuario que permite el uso de datos explícitos. Por otro lado existe escepticismo acerca del uso de datos implícitos para crear estereotipos imprecisos basados en complejos algoritmos de inferencia (Peyton, 2003), ya que una gran cantidad de de literatura reporta pocas pruebas con usuarios. "Una rápida mirada de los 9 primeros años de UMUAI revela que sólo una tercera parte de los artículos incluye algún tipo de evaluación. Esto es un porcentaje muy bajo" (Chin, 2001).

\section{8. $\mathrm{HCl}$ en las IUls}

El enfoque de HCI para resolver los problemas de interacción se ha centrado en el uso de diferentes modos de interacción o modalidades para comunicar al usuario con el sistema. La idea central es realizar esta interacción de la manera más parecida a como lo hacemos los humanos. Si tomando en cuenta que los humanos percibimos el mundo a través de los sentidos (tacto, vista, oído, olfato y gusto), parece lógico la idea de unificar esta información en una computadora que sea capaz de procesarla a través de diferentes modos de acuerdo a los dispositivos disponibles (teclado, micrófono, cámara, etc.) dando lugar a una interfaces 
multimodal. Las interfaces multimodales combinan dos o más modos diferentes de comunicación con el objeto de mejorar el canal de interacción entre el humano y el sistema. Las interfaces multimodales actuales pueden procesar dos o más modos de entrada combinados usando tecnologías basadas en reconocimiento, con el objeto de identificar con precisión o interpretar las intenciones de comunicación del usuario (Reeves et al., 2004). Existen varios tipos de sistemas multimodales, incluyendo aquellos que procesan voz y entrada manual con lápiz o a través de pantallas sensibles al tacto y audiovisual. Las tecnologías de visión computacional y procesamiento de voz son fundamentales al desarrollo de este tipo de interfaces y son revisadas extensamente en (Oviatt et al., 2000). Un enfoque novedoso en las interfaces multimodales es la integración de tecnología de agentes, componentes multimedia y de realidad virtual junto con técnicas de inteligencia artificial para desarrollar personajes similares a humanos que interactúan con los usuarios de una manera más natural. Mediante el uso de de visión computacional y procesamiento de voz, estos personajes animados o agentes conocidos como ECAS Embodied Conversational Agents representan una línea de investigación que integra varias áreas (Cassell, 2000). En estos ambientes virtuales de los ECAs, las interfaces enfrentan nuevos retos de diseño para HCI.

\section{Sistemas relacionados}

A continuación se presentan algunos de los sistemas reportados en la literatura de interfaces inteligentes que presentan algunas de las características de adaptación relevantes a la propuesta doctoral presentada en este documento.

\subsection{Sistema SurfLen}

Una de los elementos centrales de las interfaces adaptivas es su capacidad para anticipar las tareas que realizará el usuario basada en observar las acciones actuales, su comportamiento y el modelo del usuario. Una línea de investigación reportada en la literatura de IUI se basa en establecer acciones (tareas, navegación, planes, estrategias, etc.) futuras considerando el análisis del historial de las acciones pasadas del usuario (o también de un conjunto de usuarios) mediante el uso de técnicas de IA con el objeto de llevar a cabo algún tipo de razonamiento sobre dicha información e inferir la acción a seguir. El trabajo de (Fu, 2000) se ubica dentro de esta categoría, pero está enfocado a un sistema de recomendación de información basado en Web.

La hipótesis planteada por los autores es que el historial de navegación del usuario contiene información suficiente para descubrir conocimiento acerca de páginas de interés al usuario, sin la necesidad de que el usuario le asigne una calificación o tecleé información adicional, lo cual es una desventaja de los sistemas actuales. Los autores plantean que de manera activa pero silenciosa es posible mantener el seguimiento de lo que el usuario ha leído, recolectar el historial de navegación en un repositorio centralizado y aplicar técnicas de IA para descubrir conocimiento. Por ejemplo, si dos usuarios han leído varias páginas similares, podemos inferir que ambos usuarios tienen intereses similares. Este conocimiento sobre la similaridad de patrones de navegación, es usado para generar (inferir) las recomendaciones. Para probar los planteamientos, los autores desarrollaron un prototipo de un sistema de recomendación llamado SurfLen que sugiere páginas de interés a usuarios sobre tópicos específicos. El prototipo utiliza el algoritmo de Agrawal propuesto por los autores para 
generar el conjunto de reglas de asociación con el uso de una optimización apriori. La evaluación del prototipo reporta que el número de sugerencias correctas por usuario aumenta conforme el historial de navegación se vuelve más representativo de sus intereses.

La parte relevante del artículo es el uso del algoritmo de optimización A-priori de Agrawal aplicado al historial de navegación pero con el objeto de realizar recomendaciones. Asimismo el esquema propuesto con reglas de asociación ofrece ventajas sobre sistemas similares al no requerir intervención por parte del usuario. La parte débil del artículo es que las pruebas son simuladas y en un entorno controlado. En los resultados de las pruebas del prototipo se menciona que ofrece ventajas sobre otras técnicas, sin embargo no hace ninguna comparación con un sistema similar. Por otro lado, la creación de los datasets conforme se va aumentando el número de URLs y de usuarios debe resultar costoso en tiempo de procesamiento, aún con la optimización propuesta, tema que no se menciona.

El presenta artículo es relevante a a la propuesta doctoral al exponer el uso de técnicas de IA para analizar la manera en que los operadores navegan la interfaz de un sistema de supervisión y control, con el objeto de implementar el mecanismo de adaptación. Una posibilidad sería analizar el historial de navegación para operadores (experto, novato, intermedio) en situaciones (normal, emergencia) o en fases (arranque, operación, paro), para crear un patrón de navegación que me permita predecir (recomendar) el siguiente despliegue (o acción) basado en el historial de un operador (o de varios operadores similares)

\subsection{Interfaces Adaptivas para Control de Procesos}

El sistema reportado en (Viano et al., 2000) resulta relevante debido a que aborda el tema de las interfaces adaptivas, enfocado a interfaces de usuario para operaciones críticas tales como el control de procesos. Adicionalmente el prototipo presentado por los autores se plantea para ser utilizado en dos aplicaciones: un sistemas para el manejo de redes eléctricas y un sistema para la supervisión de una planta de generación termoeléctrica.

Los autores argumentan que debido a la complejidad cada vez mayor en los sistemas de control, las limitaciones de los operadores para manejar grandes cantidades de información en tiempo real y en situaciones de falla, junto con las exigencias para mantener la continuidad en la operación, permite considerar el enfoque de los sistemas adaptivos, los cuales pueden alterar su estructura, funcionalidad o interfaz con el objeto de acomodar las diferentes necesidades individuales o grupos de usuarios. El enfoque adaptivo que proponen permite asistir al operador en adquirir la información más relevante en cualquier contexto particular.

El planteamiento central del artículo es que el diseño de la interfaz del operador es rígido, es decir, se establece durante el tiempo de diseño, tomando en cuenta las mejores guías o prácticas recomendadas por $\mathrm{HCI}$, sin embargo, una vez establecido, se mantiene durante la ejecución de la aplicación. Este mapeo entre el tipo de información que se está recibiendo de campo y la forma (y medios) utilizado para presentarlo al usuario, no es única, sino que el diseñador selecciona la que considera más efectiva y eficiente, sin embargo otros posible mapeos son descartados. Así, sin importar que tan bueno sea el diseño, será fijo con las desventajas asociadas tales como una estructura rígida, no diseñado para situaciones de emergencia, etc. La arquitectura de interfaz adaptiva propuesta considera la adaptación en la presentaci' on de información. Los autores denominan su modelo como de "Mapeo 
Flexible" basado en el estado actual del proceso, el ambiente y el conocimiento de factores humanos.

El artículo propone una arquitectura multi-agentes, con agentes para las siguientes funciones: modelo del proceso, medios, resolución de despliegue, presentación, base de datos de factores humanos y operador. Asimismo se contemplan dos principios para iniciar el mecanismo de adaptación: cuando se detecte desviación del proceso y en la desviación del operador (no reacciona de acuerdo al procedimiento esperado).

$\mathrm{Al}$ inicio los autores mencionan que los beneficios del enfoque propuesto se evalúan en dos prototipos, sin embargo en la sección 6 (Prototipos), se precisa que los dos prototipos están en desarrollo no se presenta ningún tipo de evaluación. La parte de aportación de este artículo a mi trabajo de investigación es la descripción de las necesidades en los sistemas de control de procesos donde un esquema adaptivo es necesario.

La parte fuerte del artículo es la arquitectura propuesta, aún cuando la explicación dada sobre los componentes y la interacción entre ellos sea escasa. Por otra parte los autores no mencionan los mecanismos de coordinación, aspecto importante y que representa un reto en los sistemas multi-agentes. En términos generales los autores no profundizan en ningún aspecto y el estado del arte que presentan es básico.

\subsection{Sistema ADAPTS}

El sistema ADAPTS presentado por (Brusilovsky \& Cooper, 2002) aborda la utilización de diferentes modelos (tareas, usuarios y entorno) para adaptar contenido y navegación en un sistema adaptable de hiper-media. Estas características son similares a las de la propuesta doctoral, donde se plantea realizar un modelo de interfaz adaptiva de iniciativa combinada, aplicada al dominio eléctrico dentro de un sistema inteligente de ayuda con el objeto de adaptar el contenido de la información presentada, tomando en cuenta las características del usuario y el estado de la planta.

Los autores describen el sistema ADAPTS Adaptive Diagnostics And Personalized Technical Support, un sistema electrónico de soporte para técnicos de mantenimiento compuesto por una guía adaptiva a partir de un sistema de diagn' ostico con acceso adaptivo a información técnica, abarcando ambos lados del proceso: qué hacer y cómo hacerlo. Es un proyecto extenso resultado de la colaboración de investigadores de la Naval Research Airs Warfare Center, Aircraft Division, Carnegie-Mellon University, University of Connecticut y la compañía Antech Systems Inc.

Un componente importante del sistema lo constituye el IETM Interactive Electronic Technical Manuals, el cual proporciona una gran cantidad de información acerca del sistema: Cómo está construido, su operación y qué hacer en caso de problemas específicos, etc. ADAPTS es un sistema adaptivo complejo que ajusta la estrategia de diagnóstico en base a quién es el técnico y qué es lo que está haciendo, adaptando dinámicamente la secuencia de configuraciones, pruebas, procedimientos de reparación/reemplazo basado en las respuestas del técnico. Asimismo integra conocimiento del dominio, tareas de mantenimiento y las características de usuario.

El modelo del usuario propuesto por los autores determina qué tarea se debe realizar, qué información técnica seleccionar para describir la tarea y cómo presentar de la manera más adecuada dicha información para un técnico particular. La problemática planteada en el artículo es que la cantidad de información potencialmente relevante en un momento dado dentro del proceso de reparación puede llegar a ser muy grande y es un reto para los técnico 
encontrar la información más adecuada a su experiencia y contexto de trabajo. Para esto es necesaria llevar a cabo de manera permanente una valoración dinámica del conocimiento del técnico, su experiencia, preferencias y contexto de trabajo. En el modelo propuesto la experiencia es calculada a partir de varias evidencias sobre el nivel de experiencia del técnico que son recolectadas por el sistema al interactuar con el técnico. Asimismo ADAPTS utiliza un modelo de usuario tipo sobreposición multi-aspectos ( Multi-Aspect Overlay Model) el cual resulta más expresivo, pero al mismo tiempo más complejo de generar y mantener.

Los autores presenta el sistema ADAPTS, sus modelos propuestos y cómo pueden ser utilizados para construir un sistema adaptivo, sin embargo no especifican (justifican) porque los 20 aspectos propuestos utilizados para evaluar la experiencia de los usuarios son suficientes o los adecuados. Un aspecto cuestionable es que los autores asumen en su modelo que si un usuario solicita un despliegue del IETM necesariamente lo lee, sin embargo convendría incluir algún nivel de probabilidad o tiempo para establecer esta afirmación, como lo hacen otros sistemas similares ante esta situación. Llama la atención que no se presentan datos sobre experimentos de validación, su evaluación, utilidad real, aplicación en campo, satisfacción de los usuarios, etc., a pesar de que se trata de un sistema extenso y completo..

\subsection{Modelo de decisión}

El enfoque propuesto en (Stephanidis et al., 1997) presenta a las interfaces inteligentes de Usuario como componentes que se caracterizan por su capacidad de adaptarse en tiempo de ejecución y tomar varias decisiones de comunicación referente al "qué", "cuando", "por qué" y "cómo" para interactuar con el usuario, todo esto mediante el empleo de una estrategia de adaptación.

El uso de modelos como una parte central para ampliar el entendimiento sobre los procesos involucrados en la adaptación de las interfaces se presenta en (Puerta, 1998), donde se hace una clara diferencia entre un modelo y una arquitectura y los objetivos que persiguen cada uno.

Los autores conceptualizan la estrategia de adaptación como un proceso de toma de decisiones, caracterizada por atributos que involucran aspectos de la interfaz de usuario que están sujetos a adaptación y denominados por los autores como "constituyentes de adaptación". Asimismo la adaptación en tiempo de ejecución implica cierto tipo de monitoreo de la interfaz con el objeto de evaluar el estado de los elementos críticos de la interacción llamados "determinantes de adaptación" y sobre los cuales se condicionan las decisiones de adaptación. Otro aspecto abordado son los Objetivos propios del proceso de adaptación. En el esquema propuesto por los autores, se establece que las adaptaciones serán realizadas mediante un conjunto de reglas, llamadas "reglas de adaptación" que en esencia y de manera simplificada lo que hacen es asignar ciertos "“constituyentes de adaptación" a "determinantes de adaptación" específicos, dado un conjunto de "Objetivos de adaptación".

Una de las motivaciones es el hecho que aunque varios enfoques han sido reportados en la literatura, actualmente no existe consenso respecto a las características, comportamiento y componentes esenciales que deben conformar las interfaces inteligentes. El problema que plantean es que los elementos críticos del proceso de adaptación (determinantes, constituyentes, objetivos y reglas) difieren sustancialmente en los sistemas actuales. Así 
tenemos que los sistemas existentes adaptan ciertos constituyentes predefinidos, basado en un conjunto predeterminado de determinantes, a través del uso específico de reglas, con el fin de alcanzar objetivos pre-especificados. Con lo anterior argumentan que el proceso de adaptación no es flexible y que no puede ser transferido fácilmente entre aplicaciones. Para enfrentar las limitaciones anteriores los autores proponen:

1. Utilizar un enfoque metodológico que permita la personalización del conjunto de determinantes de adaptación y constituyentes de adaptaci' on.

2. La incorporación de los objetivos de adaptación como parte integral del proceso de adaptabilidad $y$.

3. la modificación de las reglas de adaptación, de acuerdo a los objetivos de adaptabilidad.

La idea central del enfoque propuesto está basada en una clara separación de los atributos, del proceso de adaptación. También se plantea que al establecer esta separación, permite que los atributos de la estrategia de adaptación puedan ser personalizados fácilmente a los requerimientos de diferentes dominios de aplicación y gruposde usuario, con lo cual podrían ser reutilizados con modificaciones menores en otras aplicaciones.

Los autores presentan una arquitectura general de una interfaz inteligente, describiendo los componentes,la interacción entre ellos y su relación con la estrategia de adaptabilidad. Asimismo se presenta de manera formal una representación de los elementos de adaptación utilizados. Concluyen el trabajo con los beneficios del enfoque propuesto, los cuales parecen ser cuestionables en varios puntos, principalmente porque no ofrecen pruebas de sus afirmaciones.

\subsection{Sistemas de Iniciativa Combinada}

Los sistemas de iniciativa combinada ha surgido como una alternativa a los esquemas de interacción que permite manejar la iniciativa desde una perspectiva más flexible, aunque más compleja.

El sistema presentado por Bunt (Bunt et al., 2004) describe la importancia del soporte adaptivo, un tema central dentro del campo de las interfaces adaptivas de iniciativa combinada (Horvitz, 1999), en las cuales es necesario que en ocasiones el sistema realice las adaptaciones de manera automática, asumiendo los respectivos problemas asociados tales como falta de control, transparencia y predictivilidad, y en otras, ofrecer los mecanismos para que el usuario mismo tome control y lleve a cabo la adaptación; sin embargo hay evidencias de que muchas veces el usuario no las realiza, y cuando lo hace, no es claro si lo hace de una manera efectiva (Jameson \& Schwarzkopf, 2002). El artículo expone la necesidad de ofrecerle a los usuarios un esquema adaptivo que les apoye en la tarea de personalización de una interfaz.

Los autores proponen una solución de iniciativa combinada, en donde si el usuario es capaz de personalizar eficientemente por si mismo, no se requiere adaptación iniciada por el sistema. En caso contrario, el sistema puede intervenir para proporcionar asistencia. Asimismo se analiza el valor de la personalización y cómo ofrecer lo que llaman soporte adaptivo (ayudar a usuarios aprovechar ventajas de una Interfaz Adaptable). Se presenta un estudio experimental y se examinan los aspectos necesarios para que los usuarios lleven a cabo una personalización efectiva de una GUI basada en menús.

Resulta interesante el análisis que se hace sobre las características de las tareas y los comportamientos de personalización que afectan el desempeño. También se realiza un 
estudio con una simulación del modelo del proceso (GOMS) basado en un modelado cognitivo que genera predicciones del desempeño del usuario. Los autores analiza con datos de prueba si vale la pena realizar la personalización de la interfaz y si realmente existen beneficios.

Para probar su trabajo, se realizan 2 experimentos exploratorios utilizando el simulador GLEAN (Baumeister et al., 2000). En el primer experimento se lleva a cabo una comparación de diferentes estrategias de personalización que varían respecto al momento de su realización, es decir cuándo son realizadas y también analizan si el "overhead" de personalizar retribuye en algo. En el segundo experimento se enfocan a comparar estrategias que difieren en términos de qué funcionalidades elige agregar el usuario a la interfaz personalizada y sus implicaciones.

Como aportación del trabajo podría considerarse el uso de un modelo GOMS que le permita simular y evaluar el impacto en la personalización tomando en cuenta de manera conjunta los factores de la estrategia de personalización basada en el momento que se realiza, la frecuencia de ejecución de las tareas, su complejidad y el nivel de experiencia del usuario.

Finalmente los resultados obtenidos de los experimentos muestran la importancia de personalizar la interfaz, y consecuentemente se fortalece la justificación de "guiar" al usuario a personalizar su interfaz como una línea importante de investigación.

\section{Discusión}

De la revisión de la literatura de IUIs presentada en este capítulo se observa que la mayoría de los sistemas analizados son aplicados a sistemas basados en Web y de oficina o de propósito general. El sistema presentado por Viano (Viano et al., 2000) representa uno de los pocos sistemas para aplicaciones críticas, tales como las de operación y control, sin embargo a diferencia del modelo que proponemos (MIA-IC), su sistema es adaptivo y es aplicable a la visualización de contenidos únicamente, no incluyendo navegación adaptable. Asimismo el sistema MIA-IC es específico a tareas poco frecuentes y críticas, nicho que ninguno de los sistemas presentado aborda.

El sistema ADAPTs (Brusilovsky \& Cooper, 2002) es similar en algunos aspectos al modelo que proponemos, ya que utiliza varios modelos para realizar la adaptación, como son un modelo de usuario, un modelo del dominio y otro de tareas similar al MIA-IC. Asimismo ADAPTs utiliza un sistema inteligente que le reporta el diagnóstico de fallas y utiliza un manual electrónico para encontrar la información que le explique al usuario cómo realizar la reparación. Esta situación parece similar a la del MIA-IC, que se conecta al sistema SAO de ayuda y realiza adaptación tanto de contenidos como de navegación.

Una diferencia importante es que ADAPTs es un sistema adaptivo, mientras que en el MIAIC proponemos un esquema de iniciativa combinada, ofreciendo mayor flexibilidad en la interacción con el usuario. Por otra parte el énfasis del sistema ADAPTs es su complejo y novedoso modelo de usuario multi-aspectos que permite de manera dinámica establecer el nivel de experiencia del usuario en todo momento. En nuestro modelo, al tener un modelo integral se busca que la información de los diferentes modelos sea el componente que nos permita hacer más preciso el proceso de adaptación, no par la cantidad de información de los diferentes modelos, sino por la información más adecuada al operador y al estado de la planta. Otra diferencia es que ADAPTs una guia adaptiva para mantenimiento y no es para situaciones poco frecuentes o críticas. 
Dado que las IUIs construyen modelos al observar el comportamiento de sus usuarios, es un reto abierto aún el generar modelos útiles mediante algoritmos que realicen un aprendizaje de manera rápida. El punto aquí no se refiere al tiempo de procesamiento en el CPU, sino al número de ejemplos de entrenamiento necesarios para generar un modelo preciso de las preferencias del usuario. La mayoría de las aplicaciones de minería de datos suponen que se tienen cantidades considerables de datos, suficientes como para inducir conocimiento preciso. En contraste, las interfaces adaptivas dependen del tiempo del usuario al utilizarlas y entonces se requiere de mecanismos de inducción que puedan proporcionar una alta precisión a partir de un conjunto pequeño de datos de entrenamiento.

Otro reto abierto es la cantidad limitada de evaluación empírica que actualmente existe para los sistemas adaptivos, por lo que más investigación es necesaria para establecer de manera medible si una interfaz adaptiva es mejor comparada a una no inteligente. Varios métodos empíricos de evaluación de usabilidad han sido utilizados y adaptados en el contexto de interfaces adaptivas, tales como entrevistas, cuestionarios, el protocolo Think Aloud (Dix at al., 2003), y otros que en esencia constituyen algunos de los métodos más usados en la validación y pruebas de los sistemas tradicionales, sin embargo su aplicabilidad a los sistemas adaptivos se considera limitado.

\section{Conclusión}

Aunque hemos presentado en este capítulo la investigación en el área de IUIs, sus retos y enfoque de solución con especial énfasis en el hecho que es un campo multidisciplinario, se observa que los tres enfoques que actualmente se utilizan con mayor éxito para enfrentar los retos de las IUIs aún muestran una falta de intercambio en los hallazgos y problemas enfrentados entre las disciplinas involucradas.

De la revisión de literatura se detecta que es necesaria una mayor integración e intercambio de ideas entre las diferentes disciplinas que conforman el área de interfaces inteligentes de usuario. Creemos que la investigación en el desarrollo de nuevos modelos de interfaz que integren componentes de varias disciplinas ayudará a encontrar soluciones diferentes, pues en general se observa que actualmente la investigación que se realiza tiende a favorecer el uso de los mismos conocimientos, técnicas y metodologías probadas y aceptados dentro de una misma disciplina, y la llamada fertilización cruzada aún es necesaria. -.

\section{Referencias}

Armentano, M.; Godoy, M. and Amandi, A. (2006) Personal assistants: Direct manipulation vs. mixed initiative interfaces. Int.J. Hum.-Comput. Stud., 64(1):27-35, 2006.

Baumeister, L.K.; John, B. E. and Byrne. M. D. (2000) A com parison of tools for building GOMs models. In CHI '00: Proceedings of the SIGCHI Conference on Human Factors in Computing Systems, ACM Press, pages 211-214, New York, NY, USA.

Birnbaum, L.; Horvitz, E.; Kurlander, D.; Lieberman, H. ; Marks, J. and Roth, S. (1997) Compelling intelligent user interfaces. How much AI? In IUI '97: Proceedings of the 2nd international conference on Intelligent user interfaces, pages 173-175, New York, NY, USA. 
Brusilovsky, P. and Cooper, D. W. (2002) Domain, task, and user models for an adaptive hypermedia performance support system. In IUI '02: Proceedings of the 7th international conference on Intelligent user interfaces, ACM Press, pages 23-30, New York, NY, USA.

Bunt, A.; Conati, C. and McGrenere, J. (2004) What role can adaptive support play in an adaptable system? In IUI '04: Proceedings of the 9th international conference on Intelligent user interfaces, ACM Press, pages 117-124, New York, NY, USA.

Carenini, G,; Smith, J. and Poole D. (2003) Towards more conversational and collaborative recommender systems. In IUI '03: Proceedings of the 8th international conference on Intelligent user interfaces, ACM Press, pages 12-18, New York, NY, USA.

Cassell, J. (2000) Embodied conversational interface agents. Commun. ACM, 43(4):70-78, USA.

Chin, D. (2001) Empirical evaluation of user models and user-adapted systems. UMUAI 01', User Modeling and User-Adapted Interaction, 11(1-2):181-194, USA.

Dix, A.; Finlay J.; Abowd G.; and Beale R. (2003) Human-computer interaction (3rd ed.). Prentice-Hall, Inc., Upper Saddle River, NJ, USA.

Eirinaki, M and Vazirgiannis, M. (2003) Web mining for web personalization. ACM Trans. Inter. Tech., 3(1):1-27, USA.

Eisenstein, J. and Rich, C. (2002). Agents and GUIs from task models. In IUI '02: Proceedings of the 7th international conference on Intelligent user interfaces, ACM Press, pages 4754, New York, NY, USA.

Franklin, D.; Budzik, J. and Hammond, K. (2002) Plan-based interfaces: keeping track of user tasks and acting to cooperate. In Proceedings of the 7th international conference on Intelligent user interfaces, ACM Press, pages 79-86, USA.

Fu, X.; Budzik, J. and Hammond, K. (2000) Mining navigation history for recommendation. In IUI '00: Proceedings of the 5th international conference on Intelligent user interfaces, ACM Press, pages 106-112, New York, NY, USA.

Hook, K. (1999) Designing and evaluating intelligent user interfaces. In IUI '99: Proceedings of the 4th international conference on Intelligent user interfaces, ACM Press,pages 5-6, New York, NY, USA.

Horvitz, E.; Breese, J.; Heckerman, D.; Hovel, D.; and Rommelse, K. (1998) The Lumiere project: Bayesian user modeling for inferring the goals and needs of software users. In Proceedings of the Fourteenth Conference on Uncertainty in Artificial Intelligence, pages 256-265, Madison, WI, USA.

Horvitz, E. (1999) Principles of mixed-initiative user interfaces. In CHI '99: Proceedings of the SIGCHI conference on Human factors in computing systems, ACM Press, pages 159166, New York, NY, USA.

Jameson A. and Schwarzkopf, E. (2002) Pros and cons of controllability: An empirical study. In AH '02: Proceedings of the Second International Conference on Adaptive Hypermedia and Adaptive Web-Based Systems, Springer-Verlag, pages 193-202, London, UK.

Kirsch-Pinheiro, M.; Villanova-Oliver, M.; Gensel, J. and Martin H. (2005) Context-aware filtering for collaborative web systems: adapting the awareness information to the user's context. In SAC '05: Proceedings of the 2005 ACM symposium on Applied computing, ACM Press, pages 1668-1673, New York, NY, USA. 
Kobsa, A. (1994) User modeling and user-adapted interaction. In CHI '94: Conference companion on Human factors in computing systems, ACM Press, pages 415-416, New York, NY, USA.

Langley, P. (1999) User modeling in adaptive interfaces. In UM '99: Proceedings of the seventh international conference on User modeling, Springer-Verlag New York, Inc., pages 357370, Secaucus, NJ, USA.

Maes, P. (1994) Agents that reduce work and information overload. Commun. ACM, 37(7):30-40, USA.

Maybury, M. (1999) Intelligent user interfaces: an introduction. In IUI '99: Proceedings of the 4th international conference on Intelligent user interfaces, ACM Press, pages 3-4, New York, NY, USA.

Mayhew, D.J. (1999) The usability engineering lifecycle: a practitioner's handbook for user interface design. Morgan Kaufmann Publishers Inc., San Francisco, CA, USA.

Mobasher, B.; Dai, H.; Luo, T. and Nakagawa, M. (2001) Effective personalization based on association rule discovery from web usage data. In WIDM '01: Proceedings of the 3rd international workshop on Web information and data management, ACM Press, pages 915, New York, NY,USA.

Oviatt, S.; Cohen,P.; Wu, L.; Vergo, J.; Duncan, L.; Suhm, B.; Bers, J.; Holzman, T.; Winograd, T.; Landay,j.; Larson, J. and Ferro, D. (2000) Designing the user interface for multimodal speech and gesture applications: State-of-the-art systems and research directions for 2000 and beyond.

Peyton, L. (2003) Measuring and managing the effectiveness of personalization. In ICEC '03: Proceedings of the 5th international conference on Electronic commerce, ACM Press, pages 220-224, New York, NY, USA.

Puerta, A. R. (1998). Supporting user-centered design of adaptive user interfaces via interface models. In Proceedings of the First Annual Workshop On Real-Time Intelligent User Interfaces For Decision Support And Information Visualization.ACM. Press. USA.

Reeves, L.M.; Lai, J.; Larson, J. A.; Oviatt, S.; Balaji, T. S.; Buisine, S.; Collings, P.; Cohen, P.; Kraal, B.; Martin, J.; McTear, M.; Raman, T.V.; Stanney, K. M.; Su, H. and Ying Q. Guidelines for multimodal user interface design. Commun. ACM, 47(1):57-59. USA.

Rich, C. and Sidner, C.L. (1996). Adding a collaborative agent to graphical user interfaces. In Proceedings of the 9th annual ACM symposium on User interface software and technology, ACM Press, pages 21-30.

Russell S. and Norvig P. (2003) Artificial Intelligence: A Modern Approach (2nd ed.). PrenticeHall, Englewood Cliffs, NJ, USA.

Shneiderman, B. and Plaisant, C. (2004) Designing the User Interface : Strategies for Effective Human-Computer Interaction (4th ed.). Addison-Wesley Publishing, Boston, USA.

Shneiderman, B. (1997) Direct manipulation for comprehensible, predictable and controllable user interfaces. In IUI '97: Proceedings of the 2nd international conference on Intelligent user interfaces, ACM Press, pages 33-39,New York, NY, USA.

Stephanidis, C.; Karagiannidis, C. and Koumpis, A. (1997) Decision making in intelligent user interfaces. In IUI '97: Proceedings of the 2nd international conference on Intelligent user interfaces, ACM Press, pages 195-202, New York, NY, USA. 
Stumpf, S.; Rajaram, V.; Li, L.; Burnett, M.; Dietterich, T.; Sullivan, E.; Drummond, R. and Herlocker, J. (2007) Toward harnessing user feedback for machine learning. In IUI '07: Proceedings of the 12th international conference on Intelligent user interfaces, ACM Press, pages 82-91, New York, NY, USA.

Sugiyama, K.; Hatano, K. and Yoshikawa, M. (2004) Adaptive web search based on user profile constructed without any effort from users. In WWW '04: Proceedings of the 13th international conference on World Wide Web, ACM Press, pages 675-684, New York, NY, USA.

Viano, G.; Parodi, A.; Alty, J.; Khalil, C.; Angulo, I.; Biglino, D.; Crampes, M.; Vaudry, C.; Daurensan, V. and Lachaud, P. (2000) Adaptive user interface for process control based on multi-agent approach. In AVI '00: Proceedings of the working conference on Advanced visual interfaces, ACM Press, pages 201-204, New York, NY, USA.

Wernn, A. (1997) Local plan recognition in direct manipulation interfaces. In Proceedings of the 2nd international conference on intelligent user interfaces, ACM Press, pages 7-14. USA. 


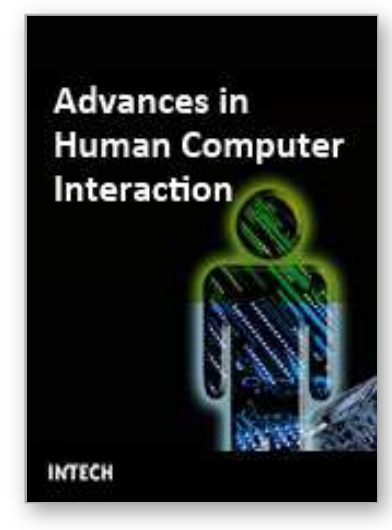

\section{Advances in Human Computer Interaction \\ Edited by Shane Pinder}

ISBN 978-953-7619-15-2

Hard cover, 600 pages

Publisher InTech

Published online 01, October, 2008

Published in print edition October, 2008

In these 34 chapters, we survey the broad disciplines that loosely inhabit the study and practice of humancomputer interaction. Our authors are passionate advocates of innovative applications, novel approaches, and modern advances in this exciting and developing field. It is our wish that the reader consider not only what our authors have written and the experimentation they have described, but also the examples they have set.

\section{How to reference}

In order to correctly reference this scholarly work, feel free to copy and paste the following:

Victor Alvarez-Cortes, Víctor H. Zarate, Jorge A. Ramirez Uresti and Benjamin E. Zayas (2008). Current Challenges and Applications for Adaptive User Interfaces, Advances in Human Computer Interaction, Shane Pinder (Ed.), ISBN: 978-953-7619-15-2, InTech, Available from:

http://www.intechopen.com/books/advances_in_human_computer_interaction/current_challenges_and_applic ations_for_adaptive_user_interfaces

\section{INTECH}

open science | open minds

\section{InTech Europe}

University Campus STeP Ri

Slavka Krautzeka 83/A

51000 Rijeka, Croatia

Phone: +385 (51) 770447

Fax: +385 (51) 686166

www.intechopen.com

\section{InTech China}

Unit 405, Office Block, Hotel Equatorial Shanghai

No.65, Yan An Road (West), Shanghai, 200040, China

中国上海市延安西路65号上海国际贵都大饭店办公楼 405 单元

Phone: +86-21-62489820

Fax: +86-21-62489821 
(C) 2008 The Author(s). Licensee IntechOpen. This chapter is distributed under the terms of the Creative Commons Attribution-NonCommercialShareAlike-3.0 License, which permits use, distribution and reproduction for non-commercial purposes, provided the original is properly cited and derivative works building on this content are distributed under the same license. 